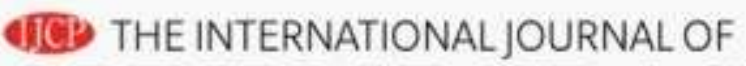 CLINICAL PRACTICE
}

\section{MANAGED CLINICAL NETWORKS: SCOPE, EVIDENCES, AND FEASIBILITY}

\begin{tabular}{|c|c|}
\hline Journal: & International Journal of Clinical Practice \\
\hline Manuscript ID: & IJCP-01-11-0014.R1 \\
\hline Wiley - Manuscript type: & Perspective \\
\hline $\begin{array}{r}\text { Date Submitted by the } \\
\text { Author: }\end{array}$ & 06-Mar-2011 \\
\hline Complete List of Authors: & $\begin{array}{l}\text { Lega, Federico; Bocconi University, Institutional Analysis and Public } \\
\text { Management } \\
\text { Sartirana, Marco; Bocconi University, Institutional Analysis and } \\
\text { Public Management }\end{array}$ \\
\hline Specialty area: & \\
\hline
\end{tabular}


MANAGED CLINICAL NETWORKS: SCOPE, EVIDENCES, AND FEASIBILITY

\author{
Keywords \\ Clinical networks \\ Cancer networks \\ Integrated care
}

\title{
Authors
}

Federico Lega, federico.lega@unibocconi.it, Bocconi University, Department of Institutional Analysis and Public Management, Via Roentgen 1, 20136, Milan, Italy.

Marco Sartirana, marco.sartirana@unibocconi.it, Bocconi University, Department of Institutional Analysis and Public Management, Via Roentgen 1, 20136, Milan, Italy.

\section{Corresponding author}

Federico Lega, Via Roentgen 1, 20136, Milan, Italy

federico.lega@unibocconi.it

tel. +393478785444

fax +390258362598 
Managed Clinical Networks (MCNs) are emerging internationally as an innovative organizational model of care for complex diseases, and are expected to achieve multiple, and in some cases contrasting, health policy goals. We provide a synthesis of expected network outcomes and discuss the evidence found in literature.ף

I

Methods

We performed a systematic search in the databases PubMed and EBSCOBusiness Source Complete, together with a large portion of grey literature on cancer MCNs. 17 relevant academic papers and grey literature documents were identified. II Results $\uparrow$

We propose the following synthesis of expected network outcomes: increase in the quality of health care services; in the equity of access; in knowledge transfer; in the cost effectiveness of health services; in patient

centeredness of services. While

intangible and intermediate outcomes were often reported, there is a paucity of evidence in favor of ultimate

network performances, especially in terms of quality of care, cost effectiveness of services and patients' experiences. ๆ ๆ

Conclusions

Our findings on cancer MCNs suggest that there are promising signs of positive returns from MCNs, yet the picture is less enthusiastic than what described in the grey literature. We discuss policy

implications and recommendations for health administrators, including the necessity to identify performance indicators and to analyze further the influence of pre-existing and contextrelated conditions on performance. 


\footnotetext{
${ }^{1}$ Intermediate tangible impacts refer to outcomes such as the changes in clinical individual activity, process development, or practice reorganization, whereas intermediate intangible impacts refer to an increase in communication, collaboration, and conflict management.
}

\section{Introduction}

Managed Clinical Networks (MCNs) are "hierarchically linked groups of health professionals and organizations from primary, secondary and tertiary care working together in a coordinated manner, unconstrained by existing professional (and organizational) boundaries to ensure equitable provision of high quality effective services" (1). The ambitious goals of MCNs include: the extension of access to care and the parallel reduction of waiting lists; the increase in the qualitative level of services; and the better use of scarce resources, including finances, technologies, and clinical expertise.

MCNs impact on multiple stakeholders - not only patients and healthcare organizations, but also health professionals, for whom network outcomes are relevant in terms of individual professional development, knowledge sharing with other professionals of the patient care pathway, multidisciplinary ways of working, improved alignment between roles and content of work, better working conditions, and more satisfaction and gratification. Many goals exist, but there is little evidence to provide guidance. With this work we shall provide a first input to fill the gap in the literature.

\section{The assessment of expected outcomes of MCNs}

We investigated the evidence provided by the literature with reference to the expected benefits of MCNs, focusing on cancer networks, which are a relevant type of MCN. We chose the case of cancer networks because they are the earliest and most significant example of MCNs, and a significant portion of the literature is consequently focused on them.

Drawing from the analysis of 17 academic contributions and grey literature documents, we propose the following synthesis of expected network outcomes ${ }^{1}$ (Table 1):

Deleted: since there are no metareviews of the effectiveness of MCNs Deleted: current

Deleted: , 
(insert Table 1)

First of all, we see how investigations on MCN performance emerge as limited in quantity and scope. In most cases, professionals' perceptions rather than objective performance outcomes were examined, and single case studies rather than comparative analyses were often performed. There is a paucity of evidence in favor of ultimate network performances, especially in terms of improving patients' experiences and increasing the quality of care.

Nevertheless, our focus on cancer networks has led to interesting findings. Beginning with the intangible impacts of cancer networks, we find (2) that cancer networks increase the formal and informal contacts among physicians, However, this trend is much less significant between GPs and hospital physicians, for it is confined to those GPs that are part of tumor groups. In general, a relevant problem of collaboration with primary care emerges $(3,4)$ Tangible intermediate outcomes are also are reported, in terms of both clinical specialization $(3,5)$ and the consequent hospital network reorganization. Moreover, collaboration in clinical practice between cancer centers and units emerges in terms of shared multidisciplinary teams, joint service planning, protocol development, and audit initiatives (3, 4, 6, 7). Improved protocol development and adoption is one of the major outcomes of networks $(2,6)$. Another relevant tangible intermediate outcome is the degree to which the network management team is provided with administrative support and controls the funding of the clinical activity. The absence of this intermediate outcome, more so than others, is usually associated with reduced ultimate network effectiveness $(4,5)$.

Moving to the analysis of ultimate network performance, the increase in quality of care is probably the most challenging dimension to be measured. If we consider the implementation of state-of-the-art, evidence-based clinical guidelines as a proxy indicator of quality of care, there is evidence that the rate of compliance with clinical guidelines is higher within a cancer network as opposed to outside of it (8). Surprisingly enough, the relationship between cancer network 
development and waiting-time reduction is scarcely addressed by existing research, raising questions of the effectiveness of MCNs in this relevant respect.

Equity is another major expected outcome of cancer networks: "networks have started to emerge as a way of sustaining vulnerable services and maintaining access where the requirements of training or subspecialization would otherwise mean complete closure of local services" (9). It was found that the establishment of a cancer network increases the access rate to specialized cancer care and clinical trials for patients living in rural areas (10). Despite the little evidence supporting the achievement of this goal, jt could be assumed $(5,7)$ that it is correlated with increased adoption of clinical pathways, increased efforts toward meeting waiting time targets (yet to be demonstrated), and increased specialization at the local level.

Results are not straightforward under the perspective of knowledge transfer, either. In some cases, network members consider MCN meetings to be an importance source of knowledgesharing regarding clinical practice, both formally and, importantly, informally (11) Paradoxically, problems emerge where informal long-standing relationships among doctors already exist and grant profitable knowledge transfer (12). With reference to these cases, which are reported in the UK, an "incompatibility between the goal of the initial technique (knowledge sharing) and the competitive nature of centralization and performance management" (11) appears. Moreover, there is a perception that cancer networks reduce the clinical expertise in cancer units in favor of cancer centers, where most complex cases are referred to $(5,11)$.

Lastly, increased cost-effectiveness - due to the achievement of economies of scale - and the reduction of transaction costs are further objectives, particularly when networks are policy lead. However, since the presence of extra resources is indicated as a key success factor for effective place to the institution of networks Deleted: these network development and management $(4,5)$, the feasibility of this goal is problematic, at least in the short run.

3. Implications and recommendations

Deleted: Therefore, MCNs are seen as the way to counterbalance the side effects of the ongoing specialization of clinical practice and facilities. There

Deleted: although

Deleted: can

Deleted: It was also found that the establishment of a cancer network

increases the access rate to

specialized cancer care and clinical trials for patients living in rural areas (10)

Deleted: In other cases, education and training activities are scarce and mostly developed by individual organizations on a specific professional basis, thus reducing the potential for knowledge-sharing.

\section{Deleted: ๆ}

Deleted: . These problems occur because the existing relationships are hampered by the development of managed networks and their performance management systems 
Although there is significant agreement over the expected outcomes of MCNs, we still do not have enough evidence to perform a thorough assessment. We have identified the following several issues concerning the impacts of $\mathrm{MCNs}_{z}$

First, we need to better understand the role played by irrational drivers. In our analysis, the focus was placed on the investigation of evidence that supports the rational-technical determinants for the creation of MCNs. However, the defining features of the context (i.e., dominant ideology, legal and institutional framework, etc.) certainly play a fundamental role in determining the drivers of networking. Public pressure represents a very influential force when officials are deciding whether to consolidate MCNs, and another irrational force for consolidation can be recognized in the fact that some leading professionals seek control over MCNs to increase their power, prestige, and benefits in the professional community Second, we should keep in mind that we need clear performance indicators allowing for the measurement of network effectiveness in terms of equity, efficiency, and clinical outcomes because, up to now, we have had a lack of significant evidence that can clearly support the expected benefits of MCNs,

Third, as MCNs are under development in different fields (e.g., cancer, diabetes, neurosciences, cardiovascular, orthopedics), it will be important in the very near future to address possible conflicts that might develop between different MCNs. There is evidence of overlapping competence zones that must be managed with collaborative approaches.

Finally, if MCNs grow in size and relevance, would it be possible and beneficial to directly allocate some resources to them for the "disease management" of some cohorts of patients? How would these fit in with current institutional boundaries set by organizations to which MCNs' members belong?

Questions for managers, policymakers, and academics are multiple, and it's extremely important to distinguish the rhetoric from the facts in order to avoid a waste of precious resources and time. The rising debate in the literature and the consolidated experiences developed in several countries show that the time may now be right for exploring the answers.
Deleted:

Deleted: First, when looking at other network experiences, the context and the limits of the studies on MCNs should be taken care of. For example, MCNs can vary significantly in size and catchment area (i.e.,

geographical coverage). Optimal size and coverage though depend on multiple variables: the demographic and geomorphologic features of the territories, the diffusion of

technologies and clinical expertise for the care of the specific disease, the complexity of the care process and the degree of required

multidisciplinarity, and the type of network that is in place. I Second

Deleted: ๆ

Third

Deleted: The relationship between the results of MCNs and their governance structure should also be considered. The same can be said for the collaboration mechanisms that

they introduce and the extent to which these are influenced by pre-existing and context-related conditions. Furthermore, there is a potential "turf war" between health system institutions/organizations and scientific societies: who is responsible for developing and governing MCNs? Lastly, there is mixed evidence with regard to professional development and the knowledge-sharing experienced by clinicians that are integrated in MCNs.

Deleted: Fourth 


\section{References}

1 The Scottish Executive, Department of Health. Introduction of managed clinical networks in Scotland. Edinburgh. 1999.

2 McCallum, A., Brommels, M. How do clinical networks and organisational approaches to integrate care impact on access to health care? IJIC/WHO 2004 Conference Proceedings. 2004.

3 Kewell, B., Hawkins, C., \& Ferlie, E.. Calman-hine reassessed: A survey of cancer network development in England, 1999-2000. Journal of Evaluation in Clinical Practice, 2002, 8(3), 303-11; discussion 299-301.

4 Lega, F., Sartirana, M., Tozzi, V. The observation of cancer clinical networks: evidences from a qualitative analysis (in Italian), Rapporto OASI, 2010. Milan, Egea.

5 Livingston, M., \& Woods, K.. Evaluation of the South East Cancer Network. 2004. Glasgow.

6 SDO. NHS of England and Wales, Service Delivery and Organization programme. Networks in health care: a comparative study of their management, impact and performance. 2010. London.

7 Livingston, M., \& Woods, K. Evaluating managed clinical networks for cancer services in Scotland. International Journal of Integrated Care. 2003.

8 Ray-Coquard, I., Philip, T., de Laroche, G., Froger, X., Suchaud, J. P., Voloch, A., et al.. A controlled "before-after" study: Impact of a clinical guidelines programme and regional cancer network organization on medical practice. British Journal of Cancer. 2002. 86(3), 313-321.

9 Edwards N. Clinical Networks. British Medical Journal, 2002, 324: 63. 
10 Brunet, J. Access to specialized cancer care and clinical trials for cancer patients from non-urban areas is facilitated by a cancer network, Journal of Clinical Oncology (ASCO Part I), 2006, Vol. 24, 18S.

11 Addicott, R., McGivern, G., \& Ferlie, E.. The distortion of a managerial technique? the case of clinical networks in UK health care. British Journal of Management, 2007, 18(1), 93-105.

12 Addicott, R., McGivern, G., \& Ferlie, E.. Networks, organizational learning and knowledge management: NHS cancer networks. Public Money \& Management, 2006, 26(2), 87-94. 
Table 1: expected outcomes of MCNs

\begin{tabular}{|l|cl|}
\hline Intermediate outcomes & $\bullet$ & Intangible \\
\hline Ultimate outcomes & $\bullet$ & Tangible \\
& $\bullet$ & Quality of care \\
& $\bullet$ & Equity of access \\
& $\bullet$ & Cost effectiveness \\
& $\bullet$ & Patient centeredness \\
\hline
\end{tabular}

